\title{
Postcorrection of Current/Noltage and Electromagnetic Force for Efficient Hysteretic Magnetic Field Analysis
}

\section{AUTHOR(S):}

Sakashita, Masaki; Nishi, Kazuya; Ito, Shumpei; Mifune, Takeshi; Matsuo, Tetsuji

\section{CITATION:}

Sakashita, Masaki ...[et al]. Postcorrection of Current/Voltage and Electromagnetic Force for Efficient Hysteretic Magnetic Field Analysis. IEEE Transactions on Magnetics 2017,

53(6): 7001104.

\section{ISSUE DATE:}

2017-06

URL:

http://hdl.handle.net/2433/243799

\section{RIGHT:}

(c) 2017 IEEE. Personal use of this material is permitted. Permission from IEEE must be obtained for all other uses, in any current or future media, including reprinting/republishing this material for advertising or promotional purposes,

creating new collective works, for resale or redistribution to servers or lists, or reuse of any copyrighted component of this work in other works.; This is not the published version. Please cite only the published version.; この論文は出版社版

でありません。引用の際には出版社版をご確認ご利用ください。 


\title{
Post-Correction of Current/Voltage and Electromagnetic Force for Efficient Hysteretic Magnetic Field Analysis
}

\author{
Masaki Sakashita ${ }^{1}$, Kazuya Nishi $^{1}$, Shumpei Ito ${ }^{1,2}$, Takeshi Mifune ${ }^{1}$, and Tetsuji Matsuo ${ }^{1}$ \\ ${ }^{1}$ Graduate School of Engineering, Kyoto University, Kyotodaigaku-katsura, Nishikyo-ku, Kyoto, 615-8074, Japan \\ ${ }^{2}$ JSPS, Kojimachi Business Center Building, 5-3-1 Kojimachi, Chiyoda-ku, Tokyo 102-0083
}

\begin{abstract}
Two post-processing methods for correcting current or voltage are proposed for a more efficient hysteretic magnetic field analysis. One method uses the instantaneous power difference whereas the other derives an equivalent resistance to the hysteretic effect. The former method gives a good post-correction of current for a voltage source. A post-correction method for electromagnetic force is also discussed, and is demonstrated to give a good approximation of this force.
\end{abstract}

Index Terms — finite element analysis, current-voltage property, electromagnetic force, play model

\section{INTRODUCTION}

$\mathrm{T}$ HE DEVELOPMENT of vector hysteresis models has enabled direct iron loss evaluation in electric machinery using a magnetic-field analysis that takes into account hysteresis directly [1]-[3]. The vector hysteresis model is also used for an iron loss evaluation in a post-processing manner [3]-[5] because the direct hysteretic analysis is still computationally expensive. After a magnetic-field analysis using a nonhysteretic property of the iron core, the post-processing method using a vector hysteresis model recalculates the magnetic field vector from the magnetic flux density obtained by the non-hysteretic analysis. So far, the recalculated magnetic field is used only to evaluate iron loss even though hysteresis may affect the voltage-current property and the electromagnetic force in the electrical machinery. This is because the post-processing method has not been sufficiently discussed in the theory.

This paper discusses the theoretical background to the postprocessing method in the presence of air gap and develops a method for post-correction of voltage/current and electromagnetic force.

\section{POST-PROCESSING METHOD}

\section{A. Background of Post-Processing Method}

For simplicity, the magnetic circuit including air gap (see Fig. 1) is discussed. The magnetic circuit is governed by $h_{a c}(B) l+v_{0} B d=N i$,

where $h_{a c}$ is the magnetic field considering the AC hysteretic property of the iron core, $l$ and $d$ are the magnetic path lengths of the iron core and air gap, respectively, and $\nu_{0}=1 / \mu_{0}$ is the reluctivity in vacuo. The electric circuit is represented as

$$
e=N S \frac{d B}{d t},
$$

where $e$ is the winding voltage and $S$ is the cross section of the iron core and air gap. In (2), the coil resistance is ignored. In general, the reluctivity of an iron core is much smaller than that of an air gap. The magnetic flux in the magnetic circuit is barely affected by the iron core and depends predominantly on the air gap. This is why iron loss is often evaluated in postprocessing after a magnetic field analysis without taking the magnetization hysteresis into account.

When the hysteretic property of the iron core is approximated by a single valued function $h_{0}(B)$ without hysteresis, the magnetic and electric circuits are governed by

$h_{0}\left(B_{0}\right) l+v_{0} B_{0} d=N i_{0}, \quad e_{0}=N S \frac{d B_{0}}{d t}$,

where $B_{0}, i_{0}$ and $e_{0}$ are respectively magnetic flux density, winding current, and winding voltage when using $h_{0}(B)$.

For the current source,

$i_{0}=i, \quad e_{0} \neq e$.

From (1), (4), and the first equation of (3),

$h_{a c}(B) l+v_{0} B d=h_{0}\left(B_{0}\right) l+v_{0} B_{0} d$

is obtained. When the reluctivity of the iron core is much smaller than that of the air gap, the first terms on both sides of (5) can be neglected. Hence, $B$ approximately equals $B_{0}$.

For the voltage source,

$e_{0}=e, \quad i_{0} \neq i$.

From (2), (6), and the second equation of (3), $B=B_{0}$ is obtained similarly as for the current source.

\section{B. Direct Method and Post-Processing Method}

In this paper, the magnetic field vector $\boldsymbol{H}$ that takes account of the AC hysteretic property is given by

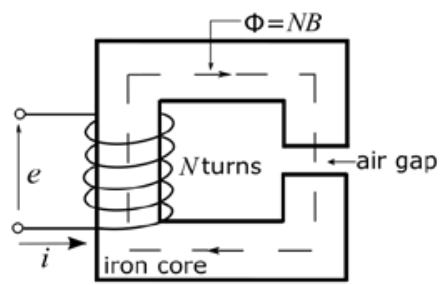

Fig. 1. Schematic of the magnetization circuit including air gap. 
$\boldsymbol{H}=\boldsymbol{h}(\boldsymbol{B})+\frac{1}{R} \frac{d \boldsymbol{B}}{d t}$,

where $\boldsymbol{h}(\boldsymbol{B})$ is a vector hysteresis function and $R$ a constant representing the AC iron loss [5], [6], for example from eddy currents.

For the current source, the FEM handling the hysteresis directly solves the differential equation,

$\operatorname{rot}\left(\boldsymbol{h}(\boldsymbol{B})+\frac{1}{R} \frac{d \boldsymbol{B}}{d t}\right)=\boldsymbol{J}$,

where $\boldsymbol{J}$ is the imposed current density vector.

When the hysteresis is taken into account by the postprocessing method, the FEM solves

$\operatorname{rot}\left(\boldsymbol{h}_{0}(\boldsymbol{B})\right)=\boldsymbol{J}$,

where $\boldsymbol{h}_{\mathbf{0}}(\boldsymbol{B})$ is a magnetic property without hysteresis. After obtaining $\boldsymbol{B}$ from (9), the magnetic field $\boldsymbol{H}$ is recalculated from (7) taking the hysteretic property into account.

For the voltage source, the electric circuit equation

$e-\frac{d \Phi}{d t}-r i=0$

is solved with (8) or (9); here $e$ is the source voltage, $i$ the current flowing in the circuit, and $r$ the coil resistance. The post-processing method is similarly applied as for the current source.

\section{Current/Voltage Post-CORREction}

\section{A. Proposed Methods to Correct Current/Voltage}

The post-processing method evaluates the iron loss including hysteresis loss using the recalculated $\boldsymbol{H}$. This section presents two post-processing methods to correct the current or voltage taking into account the hysteresis.

Using $\boldsymbol{H}_{0}=\boldsymbol{h}_{\mathbf{0}}(\boldsymbol{B})$ before post-processing and $\boldsymbol{H}$ given by (7) after post-processing, the instantaneous power consumed in the iron core is represented as

$$
\begin{aligned}
& w_{0}=e_{0} i_{0}=\int_{\text {core }} \boldsymbol{H}_{0}\left(\frac{d \boldsymbol{B}}{d t}\right) d V, \\
& w=e i=\int_{\text {core }} \boldsymbol{H}\left(\frac{d \boldsymbol{B}}{d t}\right) d V .
\end{aligned}
$$

For the current source $\left(i=i_{0}\right)$, the correction of the voltage $\delta e$ is given as

$\delta e=e-e_{0}=\frac{w-w_{0}}{i_{0}}$.

When $i_{0}=0, \delta e$ is set to 0 . For the voltage source ( $e=e_{0}$ ), the correction of voltage $\delta \mathrm{i}$ is given as

$$
\delta i=i-i_{0}=\frac{w-w_{0}}{e_{0}} \text {. }
$$

The method of correcting current or voltage by using (13) or (14) is called correction method (a) hereafter.

Corrections method (a) may yield large correction errors when $i_{0}$ or $e_{0}$ is nearly 0 . An equivalent resistance is introduced as follows. To represent iron loss for a current source, the equivalent series resistance $r_{\mathrm{a}}$ is given as $r_{a}=\delta W / I_{e}^{2}$,

where $I_{e}$ is the effective value of the current $i_{0}$ and $\delta W=\frac{1}{T} \int_{0}^{T}\left(w-w_{0}\right) d t$.

The voltage correction $\delta e$ is give as

$\delta e=i_{0} r_{a}$.

For a voltage source, an equivalent parallel conductance $g_{a}$ is defined as

$g_{a}=\delta W / E_{e}^{2}$,

where $E_{e}$ is the effective value of the voltage $e_{0}$ and $\delta W$ is given by (16). The voltage correction $\delta \mathrm{i}$ is given as

$\delta \mathrm{i}=e_{0} g_{a}$.

The method of correcting current or voltage by using (17) or (19) is called correction method (b).

\section{B. Analysis Condition}

Two types of drum-type ferrite-core inductors (Fig. 2) are analyzed using the axisymmetric 2D FEM. In this paper, the vector play model [7] is used to represent the DC hysteretic property $\boldsymbol{h}(\boldsymbol{B})$; the hysteresis-free magnetic property is given by the normal magnetization curve. The resistance $R$ in (7) is determined from the frequency dependence of coercive force [8]. The excitation frequency is $100 \mathrm{kHz}$, the coil has 35.5 turns and a resistance of $1.08 \Omega$.

\section{Comparison between Simulation and Experiment}

First, the post-processing method and the direct method are compared with the experimental result for inductor (a).

Fig. 3 presents the simulated and measured current (I)voltage $(V)$ properties with the current source. The $I-V$ curve given by the direct method roughly agrees with the measured curve. The correction to the voltage is small because the iron loss is not large.
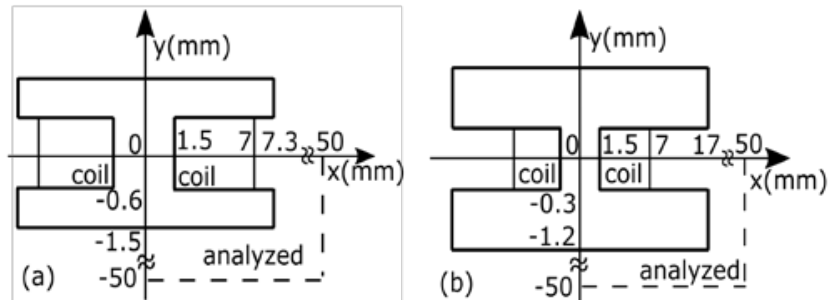

Fig. 2. Ferrite-core inductors: (a) to be compared with experiment (b) to be fed AC current or voltage including higher harmonics.

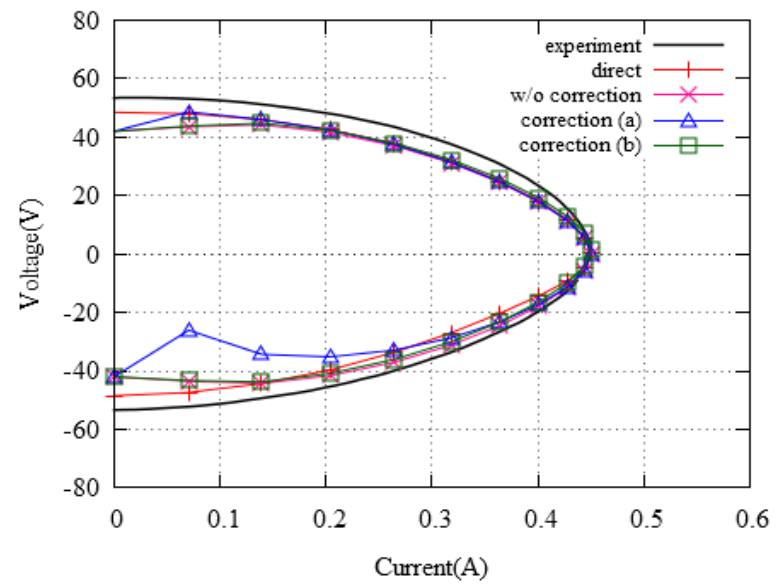

Fig. 3. Half-period of the measured and simulated current-voltage property for a sinusoidal current source. 


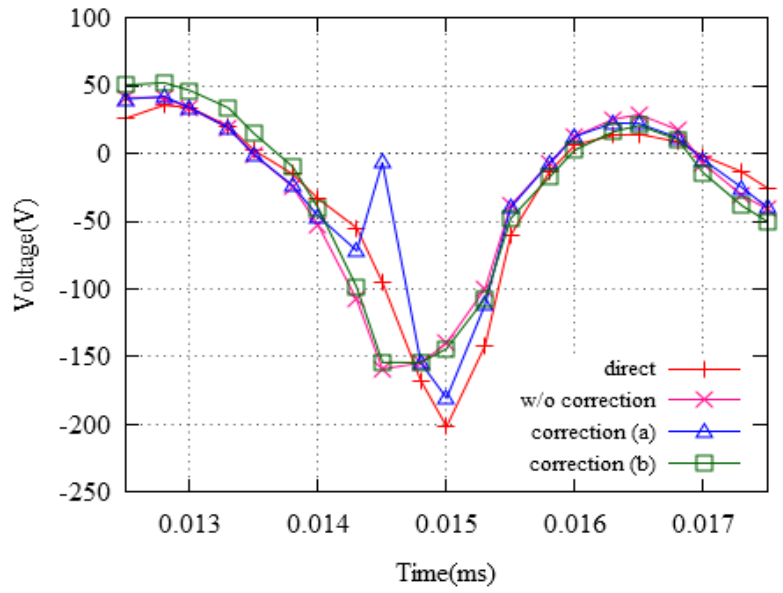

Fig. 4. Half-period of simulated voltage for current source.

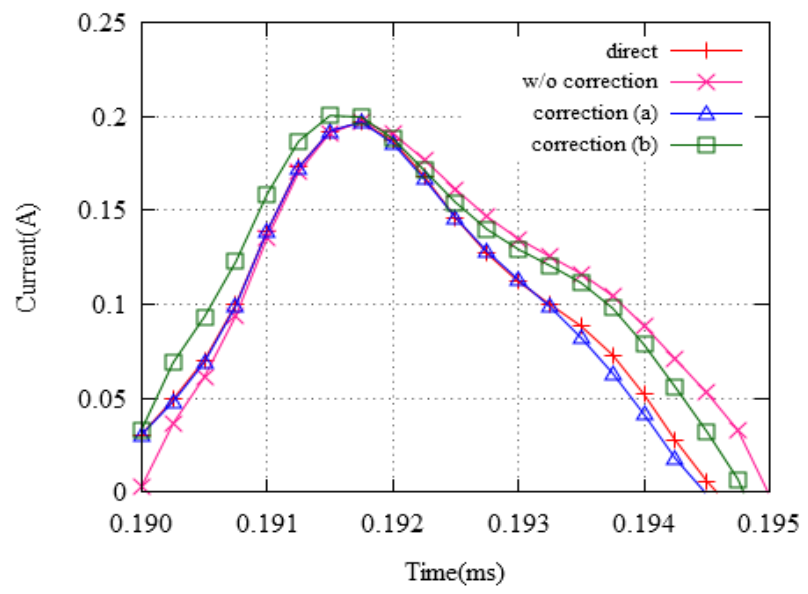

Fig. 5. Half-period of simulated current for voltage source.

\section{Comparison between Direct Method and Post- Processing Methods with Higher Harmonics}

Next, inductor (b) having large iron loss [Fig. 2(b)] is simulated to compare the post-correction methods with the direct method. The waveform of the voltage or current source includes a third-order harmonic component, specifically, $A \sin \omega t+0.3 A \sin 3 \omega t+0.3 A \cos 3 \omega t$, where $A$ is the amplitude of the fundamental component.

Fig. 4 shows the simulated voltage waveform over a halfperiod with the current source. Correction method (a) fails the voltage correction when the current is small even though $w-$ $w_{0}$ in (13) is large. This is because the recalculated magnetic field $\boldsymbol{H}$ given by the hysteretic property (7) is not small even when $i_{0}$ is nearly 0 . Correction method (b) roughly agrees with the direct method.

Fig. 5 shows the simulated current waveform over a halfperiod with the voltage source. Correction method (a) agrees with the direct method because $\mathrm{d} \boldsymbol{B} / \mathrm{d} t$ is approximately 0 when $e_{0}=0$. Correction method (b) is less accurate than correction method (a).

\section{ELECTROMAGNeTiC ForCE POST-CORRECTION}

\section{A. Method for Electromagnetic Force Correction}

The method of correcting the electromagnetic force acting

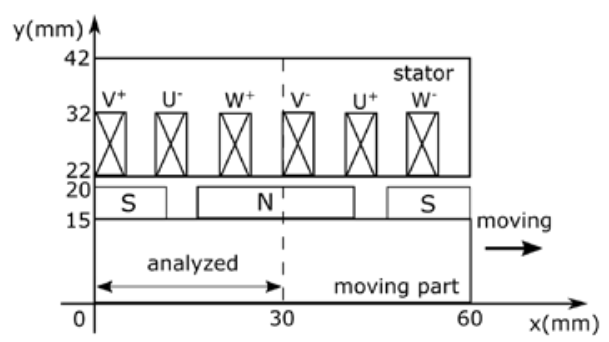

Fig. 6. 3-phase PM linear motor.
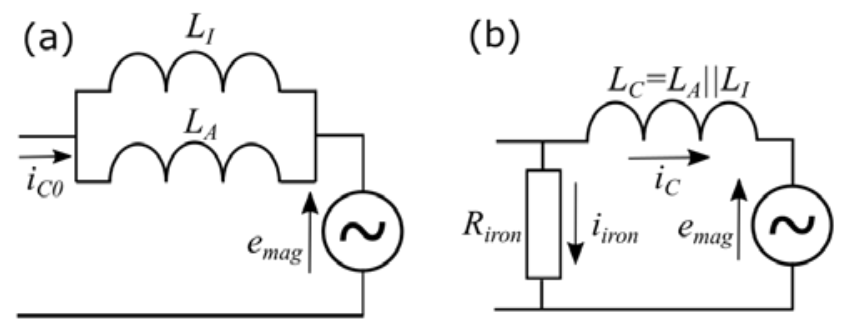

Fig. 7. An equivalent circuit of one phase of the linear motor (a) without and (b) with the hysteretic property.

on a moving part of a linear motor (Fig. 6) or a rotor of rotating machine is discussed. Denote by $P_{w}$ the work rate done by electromagnetic force $F$ in moving an object. The electromagnetic force correction $\delta F$ is given as

$\delta F=\frac{\delta P_{w}}{v}$,

where $\delta P_{w}$ is the correction to the work rate and $v$ the speed of the moved object.

Fig. 7(a) shows the single-phase equivalent circuit of a permanent magnet linear motor (Fig. 6), where $L_{\mathrm{A}}$ and $L_{\mathrm{I}}$ represent the inductances corresponding to the air gap and iron core, respectively, and $e_{m a g}$ is the approximation of the electromotive force by the magnet motion. In general, $L_{\mathrm{A}}<<$ $L_{\mathrm{I}}$ holds, which means a combined inductance $L_{\mathrm{C}}=L_{\mathrm{A}} \| L_{\mathrm{I}} \approx$ $L_{\mathrm{A}}$.

First, the post-correction for a current source $i_{0}=I \sin \omega t$ is analyzed. For simplicity, $e_{\text {mag }}$ is assumed to be

$e_{\text {mag }}=E_{M} \cos (\omega t-\theta)$,

where $E_{M}$ is the amplitude of $e_{\text {mag }}$, and $\theta$ is the phase delay to $\mathrm{di} / \mathrm{d} t$ depending on the magnet position. The input voltage $e_{0}$ is given as

$e_{0}=L_{\mathrm{C}} \frac{d i_{c 0}}{d t}+e_{\text {mag }}$

$$
=\left(\omega L_{\mathrm{C}} I+E_{M} \cos \theta\right) \cos \omega t+E_{M} \sin \theta \sin \omega t,
$$

where $i_{c 0}$ is the winding current.

When the hysteretic property $\boldsymbol{h}(\boldsymbol{B})$ is used, a parallel equivalent resistance $R_{\text {iron }}$ is introduced [Fig. 7(b)] to represent the iron loss. When $\omega L_{\mathrm{C}}<<R_{\text {iron, }}$, the input voltage $e$ approximately equals $e_{0}$. Hence, using $e_{0}, R_{\text {iron }}$ is obtained from

$R_{\text {iron }}=\frac{1}{T} \frac{\int_{0}^{T} e_{0}^{2} d t}{P_{\text {iron }}}$,

where $P_{\text {iron }}$ is the iron loss obtained in post-processing that includes hysteresis. The current flowing $R_{\text {iron }}$ is approximated as 


$$
i_{\text {iron }}=\frac{e_{0}}{R_{\text {iron }}} \text {. }
$$

which gives the winding current $i_{c}=i_{0}-i_{\text {iron. }}$. The voltage $e$ is recalculated as

$e=L_{\mathrm{C}} \frac{d\left(i_{0}-i_{\text {iron }}\right)}{d t}+e_{\text {mag }}=e_{0}-L_{\mathrm{C}} \frac{d i_{\text {iron }}}{d t}$.

From (22), (24), and (25),

$$
P_{i n}-P_{i n 0}=\frac{1}{T} \int_{0}^{T} i_{0}\left(e-e_{0}\right) d t=\frac{\omega L_{\mathrm{C}} I\left(\omega L_{\mathrm{C}} I+E_{M} \cos \theta\right)}{2 R_{\text {iron }}} \text {, }
$$

where $P_{i n 0}$ and $P_{\text {in }}$ are the input powers before and after postcorrection. From (22), $\omega L_{C} I$ and $E_{M}$ are obtained as

$$
\begin{aligned}
& E_{M}=\frac{2}{T \sin \theta} \int_{0}^{T} e_{0} \cos (\omega t) d t, \\
& \omega L_{\mathrm{C}} I=\frac{2}{T} \int_{0}^{T} e_{0} \cos (\omega t) d t-E_{M} \cos \theta .
\end{aligned}
$$

The conservation of energy gives

$\delta P_{w}=\left(P_{\text {in }}-P_{\text {in } 0}\right)-\left(P_{\text {iron }}-P_{\text {iron } 0}\right)$.

From (20), (23), and (26), $\delta F$ is obtained.

For a voltage source, $i_{c}$ is not affected by the addition of $R_{\text {iron. }}$ The electromagnetic force $F$ with hysteresis approximately equals the hysteresis-free $F_{0}$ because

$$
P_{w}=\frac{1}{T} \int_{0}^{T} i_{c} e_{\text {mag }} d t=\frac{1}{T} \int_{0}^{T} i_{c 0} e_{\text {mag }} d t=P_{w 0},
$$

where $P_{w_{0}}$ is the work rate without the hysteretic effect.

TABLE I

Simulated ENERGY AND ElECTROMAGNETIC ForCE IN THE DiRECT METHOD FOR A CURRENT SOURCE

\begin{tabular}{cccc}
\hline \hline$\theta[\mathrm{deg}]$ & 5 & 30 & 60 \\
\hline$P_{\text {in }}[\mathrm{W}]$ & 751 & 4219 & 7345 \\
$P_{\text {iron }}[\mathrm{J} / \mathrm{s}]$ & 39.7 & 38.0 & 32.3 \\
$P_{w}[\mathrm{Nm} / \mathrm{s}]$ & 712 & 4181 & 7312 \\
$F[\mathrm{~N}]$ & 237 & 1394 & 2437 \\
\hline
\end{tabular}

TABLE II

SIMULATED ENERGY AND ELECTROMAGNETIC FORCE WITHOUT CORRECTION AND WITH POST-CORRECTION FOR A CURRENT SOURCE

\begin{tabular}{cccc}
\hline \hline$\theta[\mathrm{deg}]$ & 5 & 30 & 60 \\
\hline \multicolumn{4}{c}{ without } \\
\hline$P_{\text {in } 0}[\mathrm{~W}]$ & 732 & 4198 & 7324 \\
$P_{\text {iron } 0}[\mathrm{~J} / \mathrm{s}]$ & 0.0326 & 0.318 & 0.314 \\
$P_{w 0}[\mathrm{Nm} / \mathrm{s}]$ & 732 & 4198 & 7324 \\
$F_{0}[\mathrm{~N}]$ & 244 & 1399 & 2441 \\
\hline \multicolumn{4}{c}{ post-correction } \\
\hline$P_{\text {in }}[\mathrm{W}]$ & 751 & 4217 & 7341 \\
$P_{\text {iron }}[\mathrm{J} / \mathrm{s}]$ & 41.5 & 39.6 & 33.3 \\
$P_{w}[\mathrm{Nm} / \mathrm{s}]$ & 709 & 4178 & 7307 \\
$F[\mathrm{~N}]$ & 236 & 1393 & 2436 \\
\hline
\end{tabular}

TABLE III

Simulated Electromagnetic Force For A Voltage Source

\begin{tabular}{cccc}
\hline \hline$\theta$ [deg] & 5 & 30 & 60 \\
\hline direct method [N] & 302 & 1400 & 2354 \\
without correction [N] & 302 & 1400 & 2353 \\
\hline
\end{tabular}

\section{B. Analysis of Electromagnetic Force}

A linear motor (Fig. 6) was analyzed using the 2D FEM. The magnetic field of the iron core is represented by (7) in which the second term represents the eddy-current field and $\boldsymbol{h}(\boldsymbol{B})$ originates from the vector play model [7]. The excitation frequency is $50 \mathrm{~Hz}$.

Simulated powers and electromagnetic forces are listed in Tables I and II for a current source with amplitude $I=10 \mathrm{~A}$. Post-correction gives a good approximation for the electromagnetic force and input power in the presence of hysteresis. When $\sin \theta \approx 0$, however, the correction based on (27) may become inaccurate.

Table III lists simulated electromagnetic forces for a voltage source with amplitude of $500 \mathrm{~V}$. The electromagnetic force without correction approximately equals that of the direct method as is expected from (30).

\section{CONCLUSION}

This paper proposes two methods for current/voltage postcorrection: (a) a correction using the instantaneous power difference, (b) a correction deriving an equivalent resistance. Correction method (a) achieves a good current correction for a voltage source whereas correction method (b) gives a better correction for the voltage than (a) for the current source.

The post-correction of the electromagnetic force was analyzed, indicating that force correction is not needed for a voltage source. The proposed method for a current source gives a good post-correction of the electromagnetic force and input power.

\section{REFERENCES}

[1] M. Enokizono and N. Noda, "Direct magnetic loss analysis by FEM considering vector magnetic properties," IEEE Trans. Magn., vol. 34, pp. 3008-3011, 1998.

[2] M.V.F. da Luz, J.V. Leite, A. Benabou, N. Sadowski, "Three-phase transformer modeling using a vector hysteresis model and including the eddy current and the anomalous losses,” IEEE Trans. Magn., vol. 46, pp. 3201-3204, Aug. 2010.

[3] Y. Takeda, Y. Takahashi, K. Fujiwara, A. Ahagon, T. Matsuo, "Iron loss estimation method for rotating machines taking account of hysteretic property, ” IEEE Trans. Magn., vol. 51, 7300504, Mar. 2015.

[4] J. Kitao, Y. Takada, Y. Takahashi, K. Fujiwara, A. Ahagon, and T. Matsuo, "Loss calculation method considering hysteretic property with play model in finite element magnetic field analysis," IEEE Trans. Magn., vol. 50, no. 2, pp. 381-384, Feb. 2014.

[5] S. Ito, T. Mifune, T. Matsuo, M. Suzuki, and K. Kawano, "Finite element analysis of a ferrite-core inductor with DC bias current using an equivalent-circuit model of dynamic hysteretic properties," J. Appl. Phys., vol. 115, no. 17, 17A330, April 2014.

[6] T. Matsuo, "Representation of AC hysteretic characteristics of silicon steel sheet using simple excess eddy-current loss approximation,” IEEE Trans. Magn., vol. 41, no. 5 pp. 1544-1547, May 2005.

[7] T. Matsuo, "Anisotropic vector hysteresis model using an isotropic vector play model,” IEEE Trans. Magn., vol. 46, no. 8, pp. 3041-3044, August 2010

[8] S. Ito, T. Mifune, T. Matsuo, K. Watanabe, H. Igarashi, K. Kawano, Y. Iijima, M. Suzuki, Y. Uehara, and A. Furuya, "Equivalent circuit modeling of DC and AC ferrite magnetic properties using H-Input and B-Input play models," IEEE Trans. Magn., vol. 49, no. 5 pp. 1985-1988, May 2013. 